\title{
ASSOCIATION HENRI-POINCARÉ POUR L'HISTOIRE ET LA PHILOSOPHIE DES MATHÉMATIQUES ET DE LA PHYSIQUE
}

Séminaire d'histoire des mathématiques de l'Université Paris VI Journée du 31 mars 1993

Organisée par Hourya SinACEuR

CREA Ancienne École Polytechnique

Pavillon Joffre, Amphithéâtre B

1 , rue Descartes 75005 Paris

« Théorie des modèles : aspects historiques et rapports avec les mathématiques "

$10 \mathrm{~h}$ Gabriel Sabbagh (Université Paris VII)

Théorie des groupes et théorie des modèles : quelques problèmes

11 h 15 Françoise Delon (Université Paris VII)

Logique et théorie des corps

12 h 30 Déjeuner

14 h Wilfrid Hodges (Queen Mary \& Westfield College)

Les jeux dans la théorie des modèles

15 h 15 Bruno Poizat (Universités de Karaganda et de Lyon)

Les origines de la théorie des modèles contemporaine

$16 \mathrm{~h} 30$ Pause-café

17 h Maurice Pouzet (Université de Lyon)

Sur la théorie des relations et la combinatoire

Adresse : Association Henri-Poincaré

Laboratoire de minéralogie-cristallographie

Tour 16-4, pl. Jussieu - Case 115. 75252 Paris Cedex 05.

Cotisation annuelle: normale $150 \mathrm{~F}$; de soutien $250 \mathrm{~F}$; institution $500 \mathrm{~F}$. 